\title{
Anatomía de la madera de doce especies del bosque mesófilo de montaña del Estado de México
}

\author{
Silvia Aguilar-Rodríguez ${ }^{1}$ \\ Beatriz Jimena Castro-Plata ${ }^{1}$
}

RESUMEN

\begin{abstract}
En este estudio se realizó la descripción anatómica de la madera de 12 especies propias del bosque mesófilo de montaña en el Estado de México. Para cada especie se describen los caracteres microscópicos cualitativos y cuantitativos de las maderas. Con base en la relación de Runkel se clasifica la calidad de sus fibras para obtener pulpa para papel. Se dan a conocer las características morfológicas de cada especie, así como sus nombres comunes y distribución nacional. Ocho especies tienen anillos de crecimiento formados por bandas angostas de fibras con lúmenes reducidos y paredes gruesas, o comprimidas radialmente, en la madera tardía. La porosidad es difusa; los vasos son pequeños, con menos de $100 \mu \mathrm{m}$ de diámetro y numerosos. Varias especies presentaron elementos de vaso con placa de perforación escalariforme. Los elementos de vaso y fibras se clasifican como medianos y largos. En general el parénquima axial es escaso y en siete especies los radios son de tipo heterogéneo I. Estos resultados son parecidos a los obtenidos en otras regiones similares. De acuerdo a la relación de Runkel se determinó que las fibras son de regular a buena calidad para obtener pulpa para papel.
\end{abstract}

PALABRAS CLAVE:

Anatomía de la madera, bosque mesófilo de montaña, Estado de México.

\begin{abstract}
In this study the wood anatomical description of 12 species characteristics from cloud forest in the State of México was carried out. For each species the qualitative and quantitative microscopic characters of wood are described. On the basis of the Runkel relationship, the quality of their fibers is classified to obtain pulp for paper. The morphological characteristics of each species are given, as well as their common names and national distribution. Eight species have growth rings formed by bands of fibers with lumens reduced and thick walls, or compressed radially, in the late wood. The porosity is diffuse; the vessels are small, with less than $100 \mu \mathrm{m}$ diameter, and numerous. Several species presented vessel elements with scalariform perforation plates. Vessel elements and fibers were medium and long. In general the axial parenchyma is scarce and in seven species the rays are heterogeneous type I. These results are similar to those obtained in other regions. According to the relationship of Runkel it was found out that the fibers are of medium to good quality for paper.
\end{abstract}

KEY WORDS:

Wood anatomy, Cloud forest, Estado de México. 


\section{INTRODUCCIÓN}

El bosque mesófilo de montaña (BMM) forma parte de las zonas de transición entre el clima templado y el tropical (Ortega y Castillo, 1996) y contiene un número considerable de especies arbóreas por unidad de área. Sus aproximadamente 450 especies leñosas (Rzedowski, 1996) representan un recurso potencial o real para los pobladores de comunidades rurales que se ubican en este tipo de bosque o en sus cercanías (Ortega y Castillo, 1996). De hecho, algunas especies son empleadas en la industria o localmente para diversos usos (De la Paz Pérez et al., 1982; De la Paz Pérez, 1982; Guridi y García, 1997; Ortega y Castillo, 1996; Rzedowski y Rzedowski, 1985; Torres, 1982).

En el Estado de México, el BMM ocupa menos del $3 \%$ de la entidad (Abundiz-Bonilla, 1988). No obstante, se estima la existencia de aproximadamente 200 especies leñosas (Fragoso-Ramírez, 1990; López-Pérez, 1995; Luna-Vega et al., 1989; Miranda y González, 1993; Torres-Zúñiga y Tejero-Díez, 1998) que representan un banco importante de semillas para la entidad y para otras en las que se requiera la restauración de este tipo de vegetación. El BMM se distribuye discontinuamente en la parte suroeste del Estado de México y en los declives inferiores del Ixtaccíhuatl, siendo más representativo en las cañadas que guardan suficiente humedad (FragosoRamírez, 1990; López-Pérez, 1995; Luna-Vega et al., 1989; Miranda, 1947; Miranda y González, 1993; Orozco, 1995; Torres-Zúñiga y Tejero-Díez, 1998; Rzedowski, 1986).

Tomando en cuenta su fragilidad, así como el hecho de que este bosque en el Estado de México cada vez se ve más afectado por la agricultura de temporal y la expansión demográfica (Ortega y Castillo,
1996) y que para menos de la mitad de las especies arbóreas de origen o distribución mesófila se ha reportado la descripción de su madera (Aguilar-Rodríguez et al., 2000; Barajas-Morales, 1980; Carmona, 1979; De la Paz Pérez , 1980; De la Paz Pérez, 1982; De la Paz Pérez et al., 1982; HuertaCrespo, 1975; López, 1997; Sánchez, 1968; Quintanar et al., 1996), es deseable generar la información faltante. Los estudios básicos, particularmente los referentes a la estructura anatómica de la madera, son primordiales para el avance del conocimiento taxonómico y tecnológico que requiere la entidad.

\section{OBJETIVO}

Describir los caracteres anatómicos microscópicos de la madera de 12 especies leñosas del BMM que se desarrollan en algunas regiones del Estado de México, así como proporcionar información sobre la variación anatómica entre las especies estudiadas.

\section{METODOLOGÍA}

Las muestras de madera de cada especie se colectaron en los municipios de Donato Guerra, Tejupilco y Sultepec (Tabla 1). En el campo se seleccionaron individuos maduros y de su tronco principal, a la altura de $1,30 \mathrm{~m}$, se tomaron pequeños trozos de madera de 2 a $3 \mathrm{~cm}$ de grosor empleando un serrote. En el laboratorio se obtuvieron cubos de 1,5 a $2 \mathrm{~cm}$ de la parte más cercana a la corteza, los cuales se ablandaron en glicerina-alcohol etílicoagua (GAA, 1:2:3) durante 30 días. Esta solución permite conservar contenidos celulares, en caso de que se presenten. De estos cubos se realizaron cortes de 20$30 \mu \mathrm{m}$ de grosor en los planos transversal, tangencial y radial empleando un microtomo de deslizamiento. Los cortes se tiñeron en una solución acuosa de safranina al $1 \%$ y posteriormente se sometieron 
Tabla 1. Datos de colecta de las 12 especies estudiadas ordenadas por familia

\begin{tabular}{llll}
\hline FAMILIA & NOMBRE CIENTífICO & $\begin{array}{l}\text { PROCEDENCIA } \\
\text { (MUNICIPIO) }\end{array}$ & COLECTOR/ COLECTA \\
\hline ACERACEAE & Acer negundo & Donato Guerra & D. Tejero s/n \\
CLUSIACEAE & Clusia salvinii & Tejupilco & D. Tejero s/n \\
CORNACEAE & Cornus disciflora & Sultepec & S. Aguilar 80 \\
FABACEAE & Inga spuria & Tejupilco & D. Tejero s/n \\
FAgACEAE & Quercus laurina & Sultepec & S. Aguilar 81 \\
FLACOURTIACEAE & Xylosma flexuosa & Sultepec & S. Aguilar 75 \\
MELASTOMATACEAE & Miconia glaberrima & Sultepec & S. Aguilar 78 \\
MYRSINACEAE & Myrsine juergensenii & Donato Guerra & D. Tejero s/n \\
SAXIFRAGACEAE & Phyllonoma laticuspis & Sultepec & S. Aguilar 74 \\
STYRACACEAE & Styrax argenteus & Donato Guerra & D. Tejero s/n \\
THEACEAE & Cleyera mexicana & Sultepec & S. Aguilar 76 \\
& Ternstroemia pringlei & Sultepec & S. Aguilar 79 \\
\hline
\end{tabular}

Donato Guerra: coordenadas $19^{\circ} 18^{\prime} 77^{\prime \prime}$ N. y $100^{\circ} 7^{\prime} 77^{\prime \prime}$ O; altitud 2000-2200 m; clima templado subhúmedo, precipitación media anual de $1000 \mathrm{~mm}$.

Tejupilco: coordenadas $18^{\circ} 53^{\prime} \mathrm{N}$ y $100^{\circ} 20^{\circ} \mathrm{O}$; altitud $1500 \mathrm{~m}$; clima semicálido subhúmedo, precipitación media anual de $1200 \mathrm{~mm}$, temperatura media anual de $20-22^{\circ} \mathrm{C}$

Sultepec: coordenadas $18^{\circ} 51^{\prime} 57^{\prime \prime} \mathrm{N}$ y $99^{\circ} 57^{\prime} 45^{\prime \prime} \mathrm{O}$; altitud 1900-2700 m; clima templado, el más húmedo de los subhúmedos, precipitación media anual de $1000 \mathrm{~mm}$, temperatura media anual de $14{ }^{\circ} \mathrm{C}$.

a deshidratación en alcoholes graduales y aclaración con xilol para ser montados en resina sintética (Johansen, 1940). También se disociaron astillas de madera de aproximadamente $2 \mathrm{~cm}$ de largo por $0,5 \mathrm{~cm}$ de grosor, empleando solución Jeffrey (Berlyn y Miksche, 1976). Las descripciones anatómicas se basaron en las recomendaciones de la Asociación Internacional de Anatomistas de la Madera (IAWA Committee, 1989) y para los radios en las de Kribs (1935). Tomando en cuenta la media estadística y siguiendo los criterios propuestos por Chattaway (1932) y de la IAWA (IAWA Committee, 1937, 1939), se clasificó la longitud de elementos de vaso y fibras, grosor de pared de fibras, abundancia y diámetro de vasos y abundancia y altura de radios de cada especie. De cada muestra se tomaron 50 mediciones por caracter y se obtuvieron los parámetros principales de la estadística descriptiva (media, desviación estándar y valores máximo y mínimo). Con los valores promedio de grosor de la pared de la fibra (w) y el de su lumen (L), se determinó el grado de calidad para papel de cada especie empleando la relación de Runkel, R.R. = 2w/L (Tamarit, 1996; Tomalang y Wangaard, 1961). Las especies se arreglaron en orden alfabético por familia. Además de las descripciones anatómicas, 
para cada especie, se presenta su distribución geográfica y una breve descripción morfológica basada en las especies colectadas y en sus descripciones botánicas. Entre paréntesis se señalan los datos obtenidos de los ejemplares de herbario (MEXU e IZTA) y/o bibliográficos. Los nombres científicos se citan de acuerdo con la base de datos del Missouri Botanical Garden (W3 Tropicos, 2005).

\section{RESULTADOS}

En la tabla 2 se muestra la clasificación de los elementos traqueales y radios por especie. En la tabla 3 se comparan los caracteres de algunas especies que mostraron diferencias con otros trabajos. La descripción detallada para cada taxa se presenta a continuación.

\section{Acer negundo L. (ACERACEAE) (Fig. 1 a-C).}

Características microscópicas: anillos de crecimiento conspicuos, se delimitan por una banda angosta de 4 a 5 hileras de fibras con las paredes comprimidas radialmente y pocos vasos con diámetro menor al promedio en la madera tardía. Porosidad difusa, vasos de contorno ovalado a ligeramente anguloso, principalmente solitarios y escasos múltiples radiales de 2 o 3 y hasta 5 en la madera tardía, en promedio $78 / \mathrm{mm}^{2}$, diámetro tangencial promedio de $47 \pm 3 \mu \mathrm{m}$ (34-60 $\mu \mathrm{m})$; longitud media de los elementos de vaso de $398 \pm 7 \mu \mathrm{m}(259-463 \mu \mathrm{m})$; placa de perforación simple con inclinación de 45-60P; punteaduras intervasculares poligonales alternas y ornamentadas, con diámetro de 5 a $7 \mu \mathrm{m}$; punteaduras vasoradio similares a las intervasculares; contenidos obscuros de forma esférica asociados a las paredes de los vasos. Fibras con punteaduras simples; longitud promedio de $688 \pm 10 \mu \mathrm{m}(461-813 \mu \mathrm{m})$, diámetro total de $18 \pm 2 \mu \mathrm{m}(14-22 \mu \mathrm{m})$, grosor de la pared de 3 a $4 \mu \mathrm{m}$ y diámetro del lumen de $12 \pm 2 \mu \mathrm{m}(10-14 \mu \mathrm{m})$; la relación Runkel es de 0,503 , sus fibras son de buena calidad para ser empleadas como pulpa para papel. Parénquima axial vasicéntrico, paratraqueal y apotraqueal escaso, con series de 2 a 4 células. Radios 8 /mm; tipo heterogéneo IIB; los radios uniseriados de 5 a 15 células de alto, formados por células procumbentes; los multiseriados de 3 a 4 células de ancho y pocos biseriados, formados por células procumbentes en el cuerpo y una hilera de células procumbentes de mayor dimensión o cuadradas en los márgenes, con altura promedio de $403 \pm 9 \mu \mathrm{m}$ (322$518 \mu \mathrm{m})$ y $35 \pm 5 \mu \mathrm{m}(32-43 \mu \mathrm{m}) \mathrm{de}$ ancho.

Características morfológicas: árbol de $3(2-15) \mathrm{m}$ de altura, $\mathrm{dn}^{*} 18 \mathrm{~cm}$, con ramillas densamente pubescentes, hojas opuestas, largamente pecioladas, pinnadas, inflorescencias largas colgantes; flores rojas sin pétalos; fruto alado (Martínez, 1979).

Nombres comunes: acencite, acenzitle (Méx.) icoj niquivil (Chis.).

Distribución: S.L.P., Mich., Méx., D.F., Tlax., Pue., Oax., Chis.

2. Clusia salvinii Donn. Sm. (CLusIACEAE) (Fig. $1 \mathrm{~d}$-f).

Características microscópicas: anillos de crecimiento conspicuos, se delimitan por una banda angosta de 4 a 6 hileras de fibras con paredes gruesas en la madera tardía. Porosidad difusa, vasos de contorno anguloso, principalmente solitarios y escasos en múltiples radiales de 20 3 y en racimos de $3 \circ 4$, en promedio $13 / \mathrm{mm}^{2}$, diámetro tangencial promedio de $89 \pm 7 \mu \mathrm{m}(68-112 \mu \mathrm{m})$; longitud media de los elementos de vaso de $985 \pm 26 \mu \mathrm{m}$ (706-1217 $\mu \mathrm{m})$; placa de perforación

\footnotetext{
Se entiende el diámetro a 1,30 m del suelo; antes d.a.p.
} 


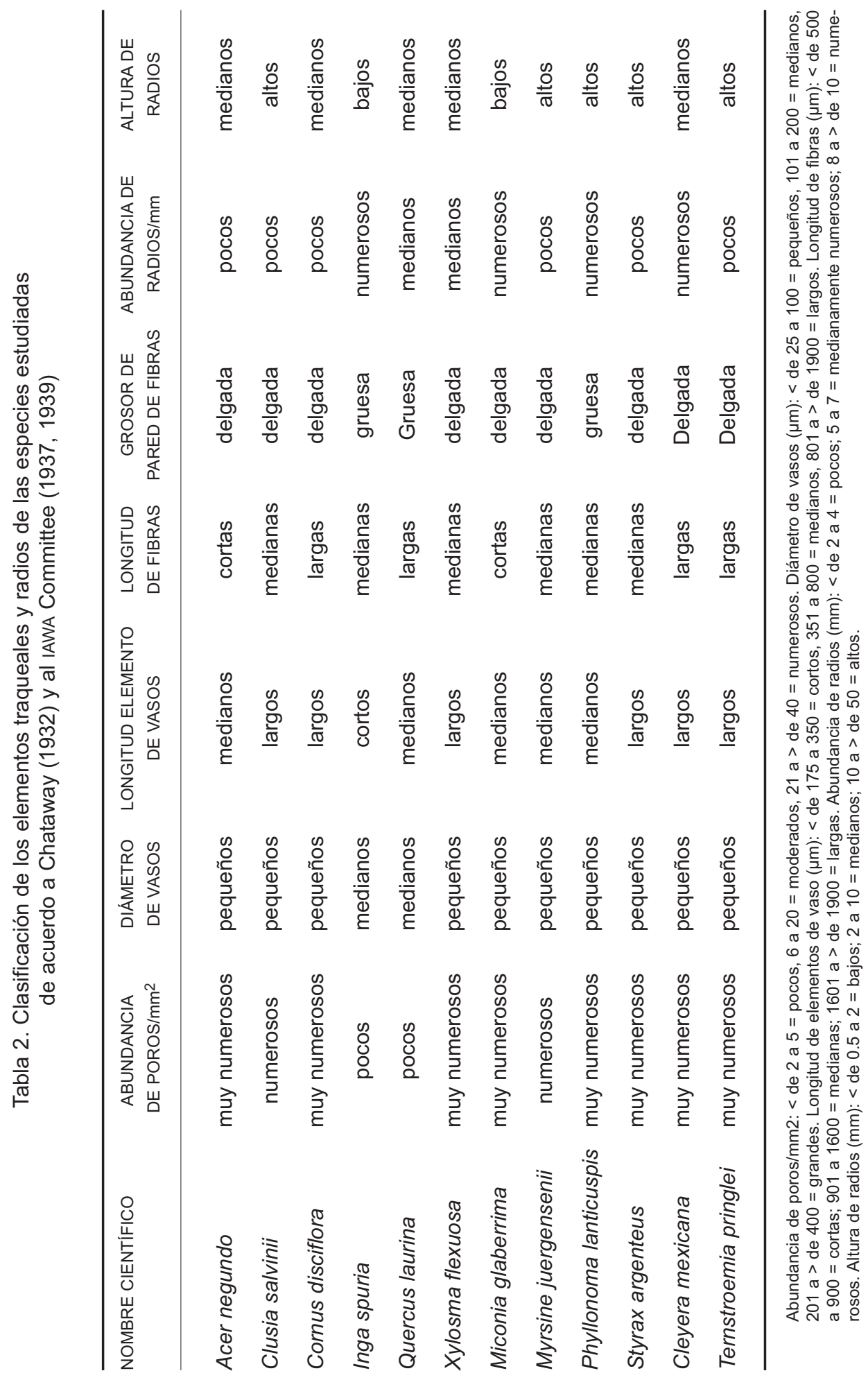


Tabla 3. Características anatómicas de algunas especies que mostraron diferencias con los datos publicados por 1. López (1997), 2. Aguilar-Rodríguez et al. (2000),

3. De la Paz Pérez et al. (1982) y 4. Quintanar et al. (1996)

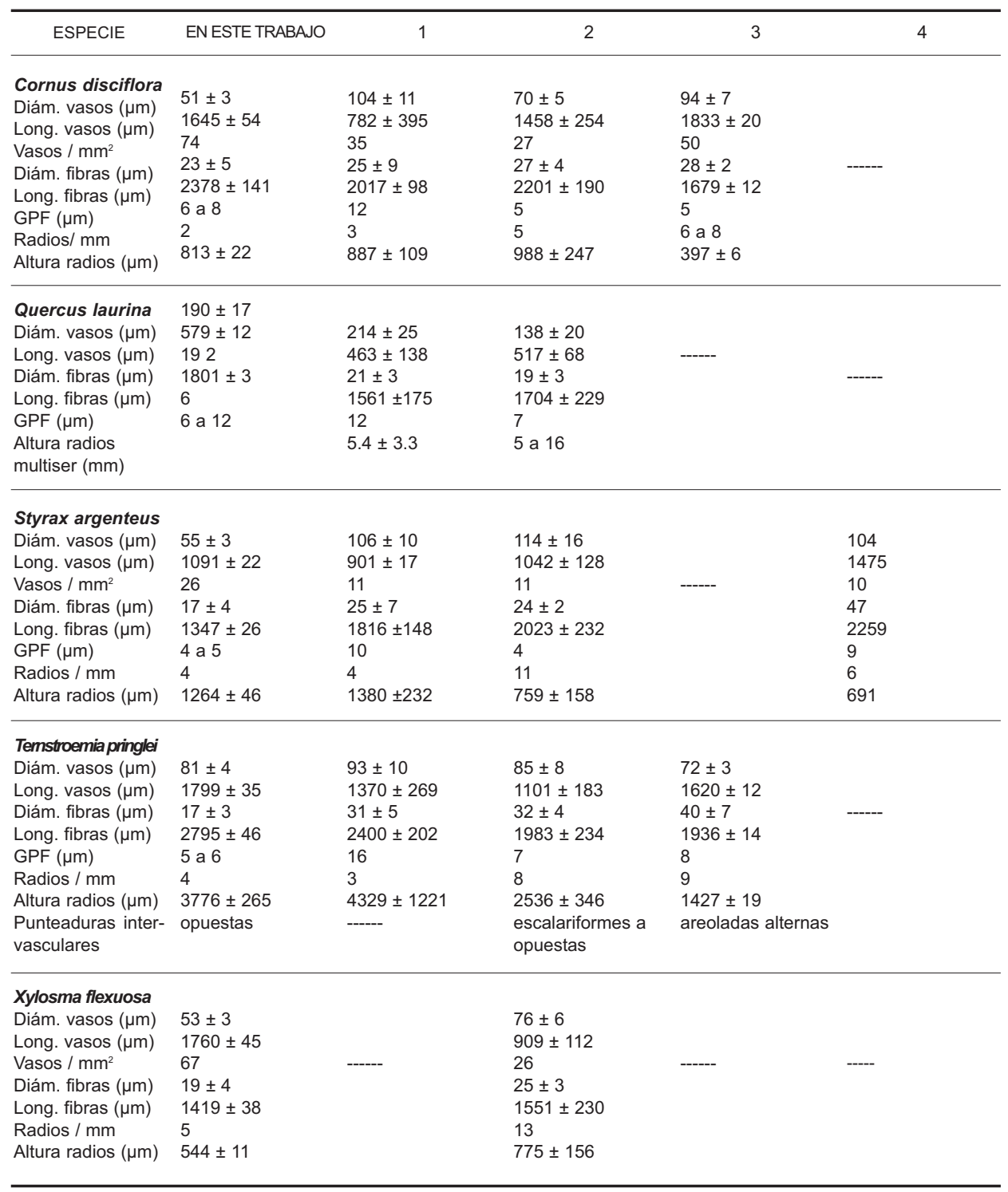


simple con inclinación de 50-60 punteaduras intervasculares escalariformes, con diámetro de 24 a $30 \mu \mathrm{m}$; punteaduras vaso-radio similares a las intervasculares. Fibras con punteaduras areoladas pequeñas, con 1 a 2 septos; longitud promedio de $1108 \pm 22 \mu \mathrm{m}(824-1327 \mu \mathrm{m})$, diámetro total de $23 \pm 4 \mu \mathrm{m}(16-30 \mu \mathrm{m})$, grosor de la pared de 8 a $9 \mu \mathrm{m}$ y diámetro del lumen de $12 \pm 4 \mu \mathrm{m}$ (7-18 $\mu \mathrm{m})$; la relación Runkel es de 0.803 , sus fibras son de buena calidad para ser empleadas como pulpa para papel. Parénquima axial paratraqueal escaso y vasicéntrico formando una vaina estrecha alrededor de los vasos, con series de 4 a 8 células; con drusas escasas. Radios $4 / \mathrm{mm}$; tipo heterogéneo I; los radios uniseriados de 3 a 4 células de alto, formados por células erectas; los multiseriados de 3 a 4 células de ancho, formados por células procumbentes y/o cuadradas en el cuerpo y de 2 a 4 , excepcionalmente 6 , hileras de células erectas en los márgenes, con altura promedio de $1321 \pm 38 \mu \mathrm{m}(863-2041 \mu \mathrm{m})$ y $69 \pm 14 \mu \mathrm{m}$ (54-97 $\mu \mathrm{m})$ de ancho.

Características morfológicas: árbol y a veces arbusto de 3 a $12 \mathrm{~m}$ de altura, en ocasiones crecen como epífitas; corteza lisa; hojas oblongas-obovadas u ovalobovadas, con nervaduras numerosas; flores de aproximadamente $10 \mathrm{~mm}$; frutos globosos. Perennifolio (Martínez, 1979).

Nombres comunes: flor de canela, oreja de venado, flor de venado, guayabillo, guicho, huicho, lengua de tigre (Mich.), palo de águila, mata palo (Oax.), oreja de tigre (Gro.).

Distribución: Nay., Jal., Ver., Col., Mich., Méx., Gro., Oax., Chis., Yuc., Q. Roo.

3. Cornus disciflora DC. (CORnACEAE) (Fig. $1 \mathrm{~g}-\mathrm{i}$ ).

Características microscópicas: anillos de crecimiento conspicuos, se delimitan por una banda angosta de 5 a 6 hileras de fibras con paredes gruesas y lúmenes pequeños en la madera tardía. Porosidad difusa, vasos de contorno ligeramente anguloso, principalmente solitarios y escasos en grupos de 2 o 3, en promedio $74 / \mathrm{mm}^{2}$, diámetro tangencial promedio de $51 \pm 3 \mu \mathrm{m}$ (38-66 $\mu \mathrm{m})$; longitud media de los elementos de vaso de $1645 \pm 54 \mu \mathrm{m}$ (1036-2041 $\mu \mathrm{m})$; placa de perforación escalariforme de 40 a 50 barras, con una inclinación de $60^{\circ}$; punteaduras intervasculares opuestas a escalariformes; punteaduras vaso-radio alternas con las areolas muy reducidas. Fibras con punteaduras areoladas evidentes; longitud promedio de $2378 \pm 141 \mu \mathrm{m}$ (1436-2967 $\mu \mathrm{m})$, diámetro total de $23 \pm 5$ $\mu \mathrm{m}(17-29 \mu \mathrm{m})$ grosor de la pared de 6 a $8 \mu \mathrm{m}$ y diámetro del lumen de $10 \pm 4 \mu \mathrm{m}$ (5-18 $\mu \mathrm{m})$; la relación Runkel es de 1.180, sus fibras son de calidad regular para ser empleadas como pulpa para papel. Parénquima axial paratraqueal escaso y apotraqueal difuso, con series de más de 8 células. Radios $2 / \mathrm{mm}$; tipo heterogéneo I; los radios uniseriados numerosos de 4 a 6 células de alto, formados por células erectas; los multiseriados de 3 a 4 células de ancho, formados por células procumbentes en el cuerpo y de 2 a 4, excepcionalmente 6 , hileras de células erectas y/o cuadradas en los márgenes, con altura promedio de $813 \pm 22 \mu \mathrm{m}$ (494-1036 $\mu \mathrm{m})$ y $38 \pm 3 \mu \mathrm{m}(31-44 \mu \mathrm{m})$ de ancho.

Características morfológicas: árbol o a veces arbusto de 15 (10-30) m de altura, dn $60 \mathrm{~cm}$, ramificación simpódica, rama en disposición ascendente (AguilarRodríguez et al., 2000); hojas opuestas oblongo-elípticas, casi blancas de abajo; flores en cabezuelas pedunculadas; copa alta esférica; fruto elipsoide color morado (Martínez, 1979). Semicaducifolio.

Nombres comunes: akiniri (Chih.), abiodo, canelo, palo canelo, isimac (Chis.), asintla, cordoncilo (Gro.), mimbre pasilla, 
pasilla blanco (S.L.P.), aceituno, guardalagua, limoncillo, mimbre prieto, palo verde, pasilla blanca, pasilla negra, variador, cinturilla, tchacari-huaní, granadillo (Mich.), flor de canastilla (Ver.), asintla (Nay.), azulillo (Jal.), mathahuacal prieto, palo pachón (Hgo.), aceituna (Méx.).
Distribución: Son., Chih., Tamps., Sin., Dgo., Zac., S.L.P., Nay., Jal., Gto., Qro., Hgo., Ver., Mich., Méx., D.F., Mor., Gro., Pue., Oax., Chis.
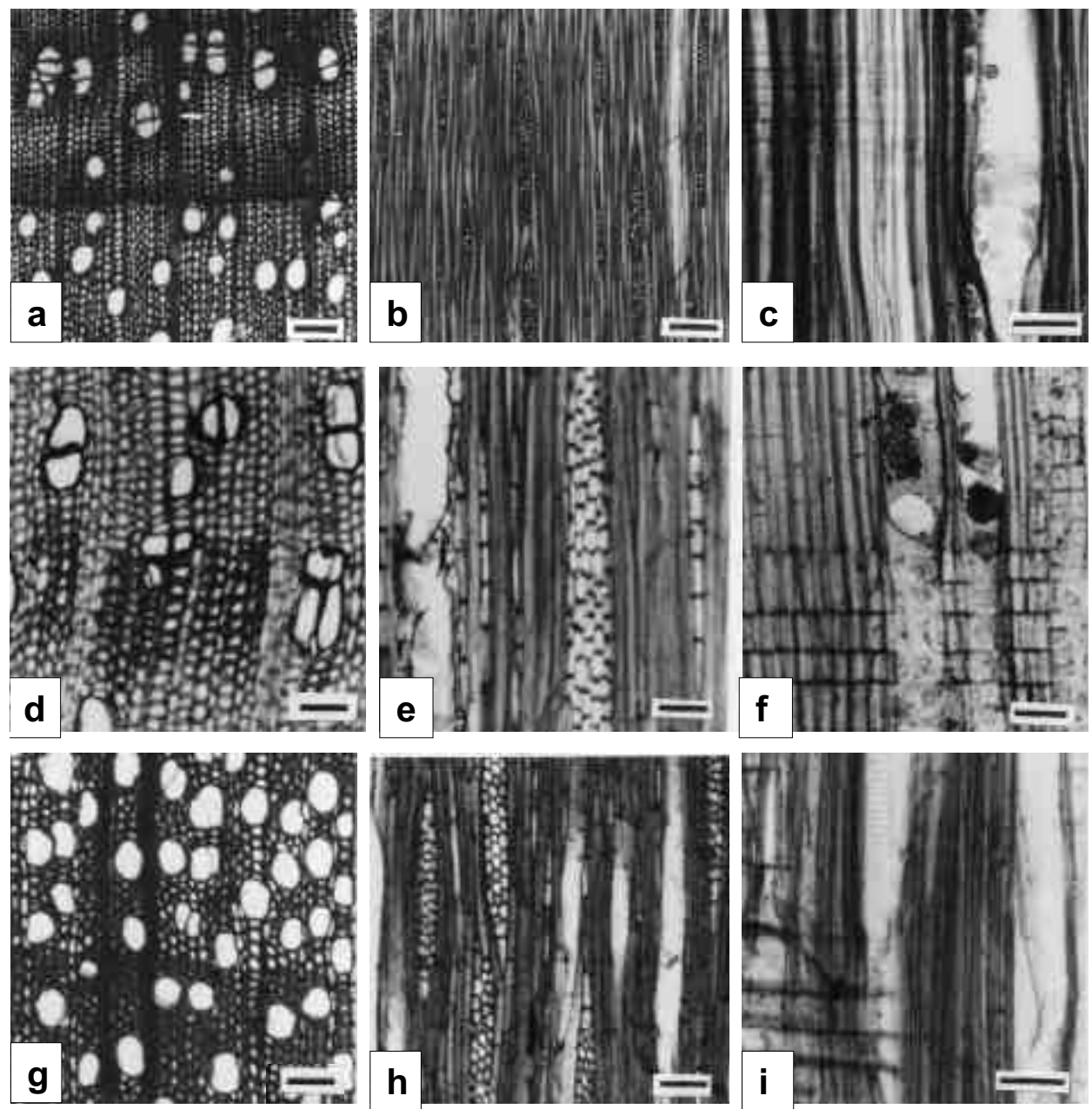

Figura 1. Características microscópicas de la madera.

Cortes transversal (izquierda), tangencial (centro) y radial (derecha). a-c. Acer negundo. d-f. Clusia salvinii. g-1. Cornus disciflora.

(escalas: $a-h=100, m-1=50 \mathrm{~m}$ ) 
4. Inga spuria Humb. \& Bonpl. ex Willd. (FABACEAE) (Fig. 2 a-d).

Características microscópicas: anillos de crecimiento conspicuos, se delimitan por una banda angosta de 1 a 2 hileras de fibras con las paredes comprimidas radialmente y parénquima marginal cristalífero en la madera tardía. Porosidad difusa, vasos de contorno circular, principalmente solitarios y escasos múltiples radiales de 2 o 3 , en promedio $4 / \mathrm{mm}^{2}$, diámetro tangencial promedio de $146 \pm 12$ $\mu \mathrm{m}(102-188 \mu \mathrm{m})$; longitud media de los elementos de vaso de $327 \pm 6 \mu \mathrm{m}$ (259$455 \mu \mathrm{m}$ ); placa de perforación simple con inclinación de $30-35^{\circ}$; punteaduras intervasculares poligonales alternas, con diámetro de 6 a $7 \mu \mathrm{m}$; punteaduras vasoradio similares a las intervasculares. Fibras con punteaduras simples, con un septo; longitud promedio de $1211 \pm 39 \mu \mathrm{m}$ (628-1546 $\mu \mathrm{m})$, diámetro total de $12 \pm 2$ $\mu \mathrm{m}(11-16 \mu \mathrm{m})$, grosor de la pared de 5 a $6 \mu \mathrm{m}$ y diámetro del lumen de $2 \pm 1 \mu \mathrm{m}$ (1$5 \mu \mathrm{m})$; la relación Runkel es de 4,220 , sus fibras son de mala calidad para ser empleadas como pulpa para papel. Parénquima axial paratraqueal aliforme y aliforme confluente, apotraqueal escaso, con series de 2 a 3 células; con largas cadenas de cristales prismáticos en cámaras. Radios $12 / \mathrm{mm}$; tipo homogéneo I; los radios uniseriados de 6 a 10 células de alto, formados por células procumbentes; los multiseriados principalmente de 2 y pocos de 3 células de ancho, formados exclusivamente por células procumbentes, con altura promedio de $169 \pm 3 \mu \mathrm{m}(126-220 \mu \mathrm{m})$ y $22 \pm 4 \mu \mathrm{m}(16-$ $29 \mu \mathrm{m})$ de ancho.

Características morfológicas: árbol de 10 (4-20) m de altura, copa amplia; hojas alternas con raquis alado, con 5 a 7 pares de hojuelas oblongas; flores blancas en cabezuelas; fruto pubescente. Perennifolio (Martínez, 1979).
Nombres comunes: agotope (Oax.), bitze, guatope (Tab.), coctzan, cuil de agua, chelel, sam, tzan (Chis.), cuaj nicuil (Mor.), chelele, timbre, vainillo, aguetope, ajotopi (Ver.), jinicuil, jinicuile, ixe Erinia tsidi (Gro.), jacanicuii (Mich.).

Distribución: Tamps., S.L.P., Jal., Col., Ver., Mich., Méx., Gro., Pue., Oax., Tab., Chis., Yuc.

\section{Quercus laurina Bonpl. (FAGACEAE)} (Fig. 2 e-g).

Características microscópicas: anillos de crecimiento inconspicuos. Porosidad difusa a semianular, vasos de contorno circular, principalmente solitarios, en promedio $5 / \mathrm{mm}^{2}$, diámetro tangencial promedio de $190 \pm 17 \mu \mathrm{m}(142-280 \mu \mathrm{m})$; longitud media de los elementos de vaso de $579 \pm 12 \mu \mathrm{m}(400-651 \mu \mathrm{m})$; placa de perforación simple con inclinación de $40-45^{\circ}$; punteaduras intervasculares alternas, con un diámetro de 7 a 9 $\mu \mathrm{m}$; punteaduras vaso-radio con punteaduras verticales; tílides ocluyen algunos vasos. Fibras con punteaduras simples; longitud promedio de $1801 \pm 33 \mu \mathrm{m}$ $(1468-2800 \mu \mathrm{m})$, diámetro total de $19 \pm$ $2 \mu \mathrm{m}(16-22 \mu \mathrm{m})$, grosor de la pared de 5 a $6 \mu \mathrm{m}$ y diámetro del lumen de $8 \pm 2$ $\mu \mathrm{m}(6-10 \mu \mathrm{m})$; la relación Runkel es de 1,112 , sus fibras son de calidad regular para ser empleadas como pulpa para papel. Se observan traqueidas vasicéntricas. Parénquima axial vasicéntrico abundante y escalariforme en bandas angostas de 1 a 3 células de ancho, apotraqueal difuso en agregados, con series de 4 a 8 células. Radios $6 / \mathrm{mm}$; tipo homogéneo I; los radios uniseriados de 5 a 17 células de alto, formados por células procumbentes; los multiseriados de 20 a 24 células de ancho, formados por células procumbentes, con altura promedio de 6 a $12 \mathrm{~mm}$ y $328 \pm 52 \mu \mathrm{m}$ (216-410 $\mu \mathrm{m})$ de ancho. 
Características morfológicas: árbol de 6 (10-30) m de altura, dn $16 \mathrm{~cm}$, tronco cónico, circular en corte transversal, copa en forma de casquete esférico, ramificación monopódica con ramas en disposición semiascendente y difusa (Aguilar-Rodríguez et al., 2000); fruto solitario o por pares; bellota ovoide. Subperennifolio (Martínez, 1979).

Nombres comunes: ahuaceptizahuac, encino laurelillo, capulincillo, tuxhi, zu-chi (Méx.), encino ahualpitzahual (Ver.), encino jarilla (Gto.), encino lucerillo (Hgo.), encino nechilahue (Pue.).

Distribución: Zac., Jal., Mich., Gto., Qro., Hgo., Pue., Méx., Mor., D.F., Tlax., Gro., Oax., Ver.

\section{Xylosma flexuosa (Kunth) Hemsl. (FLACOURTIACEAE) (Fig. $2 \mathrm{~h}-\mathrm{j}$ ).}

Características microscópicas: anillos de crecimiento conspicuos, se delimitan por una banda angosta de 3 a 5 hileras de fibras con las paredes comprimidas radialmente en la madera tardía. Porosidad difusa, vasos de contorno ligeramente anguloso, principalmente solitarios $y$ escasos múltiples radiales de 2 o 3 , en promedio $67 / \mathrm{mm}^{2}$, diámetro tangencial promedio de $53 \pm 3 \mu \mathrm{m}(42-64 \mu \mathrm{m})$; longitud media de los elementos de vaso de $1760 \pm 45 \mu \mathrm{m}(1177-2386 \mu \mathrm{m})$; placa de perforación simple con inclinación de 40-50P; punteaduras intervasculares alternas, con diámetro de 6 a $7 \mu \mathrm{m}$; punteaduras vaso-radio similares a las intervasculares; tílides ocluyen algunos vasos. Fibras con punteaduras simples, con 3 a 5 septos y con contenido amorfo; longitud promedio de $1419 \pm 38 \mu \mathrm{m}$ (1107-1672 $\mu \mathrm{m})$, diámetro total de $19 \pm 4 \mu \mathrm{m}(14-25$ $\mu \mathrm{m})$, grosor de la pared de $4-5 \mu \mathrm{m}$ y diámetro del lumen de $9 \pm 2 \mu \mathrm{m}(6-14 \mu \mathrm{m})$; la relación Runkel es de 0,856 , sus fibras son de buena calidad para ser empleadas como pulpa para papel. Parénquima axial vasicéntrico escaso, con series de 5 a 8 células. Radios $5 / \mathrm{mm}$; tipo heterogéneo I; los radios uniseriados de 4 a 6 células de alto, formados por células erectas; los multiseriados de 3 a 4 células de ancho, formados por células procumbentes en el cuerpo y con más de cuatro hileras de células erectas y/o cuadradas en los márgenes, frecuentemente los radios se continúan entre sí en sus márgenes uniseriados, con altura promedio de $544 \pm 11$ $\mu \mathrm{m}(377-706 \mu \mathrm{m})$ y $40 \pm 6 \mu \mathrm{m}(31-52 \mu \mathrm{m})$ de ancho. Algunas células de los radios presentan contenido obscuro.

Características morfológicas: árbol a veces arbusto de 5 (2-12) $m$ de altura, dn $11 \mathrm{~cm}$, tronco cilíndrico con espinas; ramas simpodiales; hojas alternas, elípticas u obovadas; flores pequeñas; fruto subgloboso. Subperennifolio (AguilarRodríguez et al., 2000).

Nombres comunes: hichichiltemel (S.L.P.), abrojo (Méx.), coronilla (Oax.), palo de brujo, yisimbolon (Chis.), granadillo, granjeno, tejocotillo (Mich.), acahual, manzanillo (Ver.).

Distribución: Son., N.L., Tamps, Sin., Dgo., S.L.P., Nay., Jal., Col., Mich., Qro, Hgo., Ver., Méx., Gro, Mor., Pue., Oax., Tab., Chis., Camp., Yuc.

\section{Miconia glaberrima (Schltdl.) Naudin. (Melastomataceae) (Fig. 3 a-c).}

Características microscópicas: anillos de crecimiento inconspicuos. Porosidad difusa, vasos de contorno ligeramente anguloso, principalmente solitarios y múltiples radiales de 2 a 4 , pocos en racimos de 4 a 6 vasos, en promedio $25 / \mathrm{mm}^{2}$, diámetro tangencial promedio de $72 \pm 6$ $\mu \mathrm{m}$ (52-92 $\mu \mathrm{m})$; longitud media de los elementos de vaso de $357 \pm 13 \mu \mathrm{m}$ (188$557 \mu \mathrm{m})$; placa de perforación simple con inclinación de $45-50^{\circ}$; punteaduras intervasculares poligonales alternas, con diámetro de 6 a $7 \mu \mathrm{m}$; punteaduras vaso- 

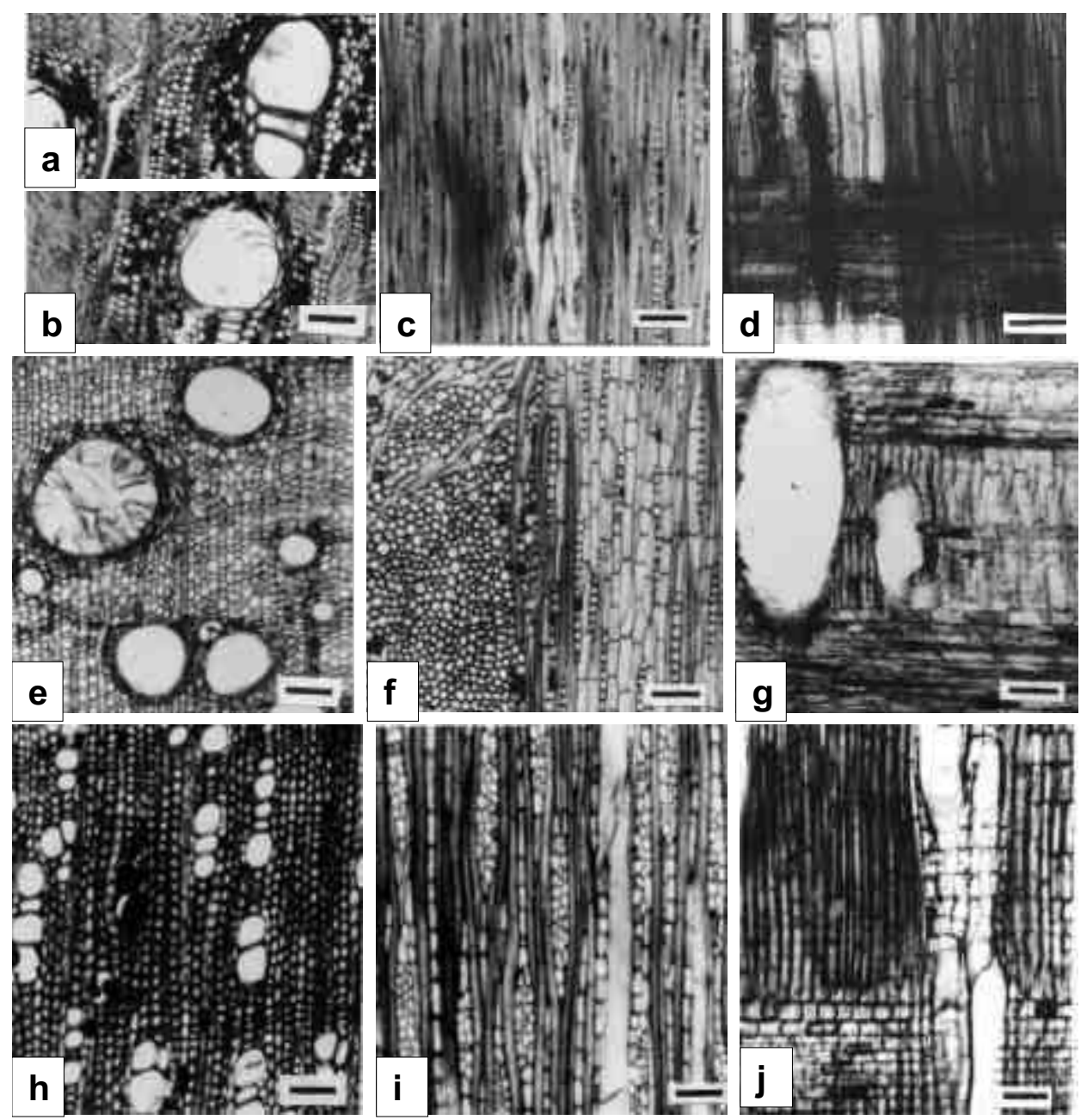

Figura 2. Características microscópicas de la madera.

Cortes transversal (izquierda), tangencial (centro) y radial (derecha). a-d. Inga spuria. e-g. Quercus laurica. h-j. Xylosma flexuosa.

(escalas: $a-c, e-j=100, m, d=50 \mathrm{~m}$ )

radio similares a las intervasculares. Fibras con punteaduras simples; longitud promedio de $523 \pm 15 \mu \mathrm{m}(385-683 \mu \mathrm{m})$, diámetro total de $16 \pm 3 \mu \mathrm{m}(13-20 \mu \mathrm{m})$, grosor de la pared de 3 a $4 \mu \mathrm{m}$ y diámetro del lumen de $10 \pm 3 \mu \mathrm{m}(7-15 \mu \mathrm{m})$, con pequeños cuerpos obscuros de forma irregular; la relación Runkel es de 0,582, sus fibras son de buena calidad para ser empleadas como pulpa para papel. Parénquima axial vasicéntrico escaso y apotraqueal difuso, con series de 2 a 3 células. Radios 13/mm; tipo heterogéneo III; radios principalmente uniseriados de 7 a más de 20 células de alto, formados por células procumbentes, cuadradas y erectas 

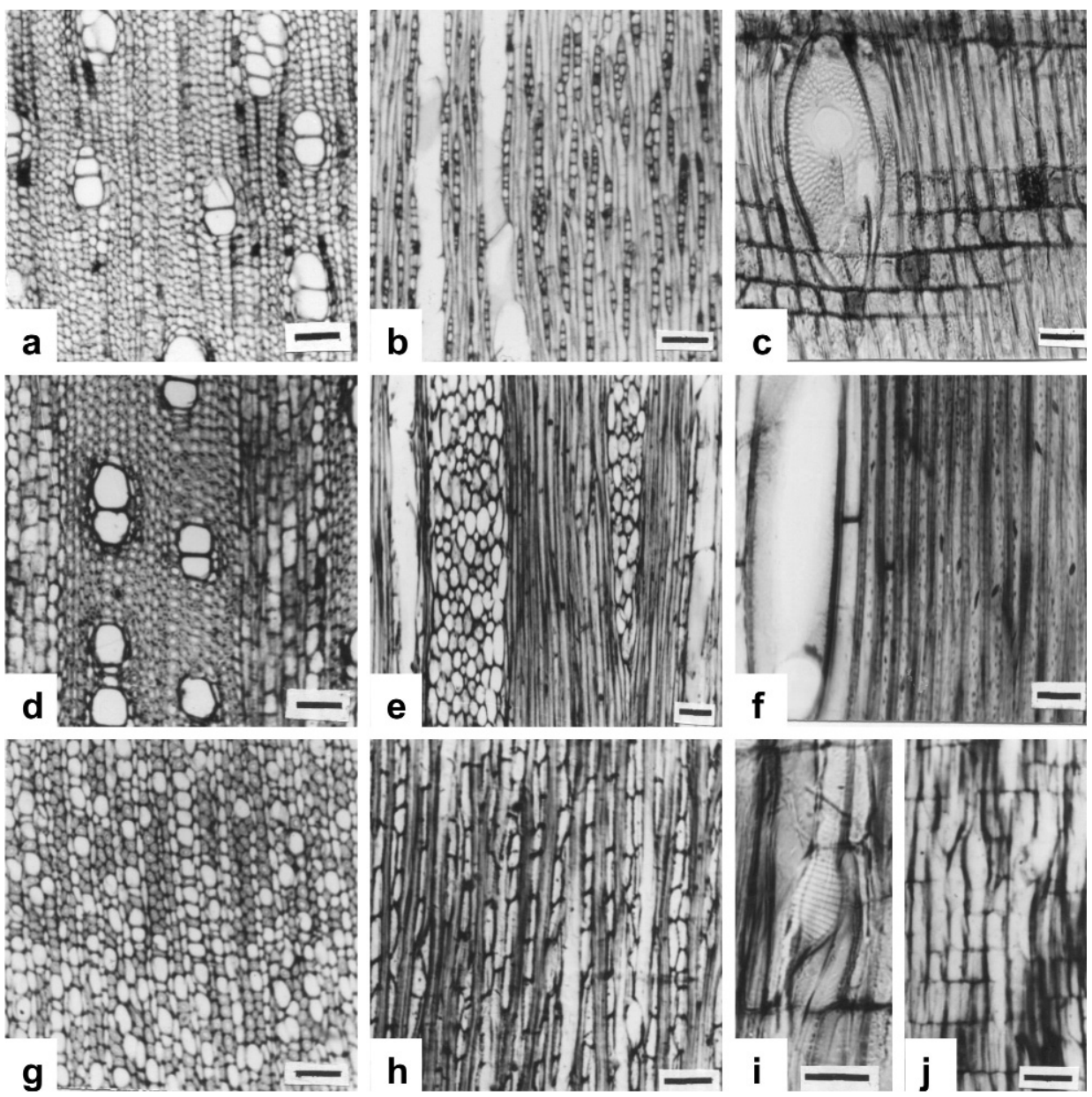

Figura 3. Características microscópicas de la madera.

Cortes transversal (izquierda), tangencial (centro) y radial (derecha).

a-c. Miconia glaberrima. d-f. Myrsine juergensenii. g-j. Phyllonoma lanticuspis.

(escalas: $a-h, j=100, \mu \mathrm{m}, \mathrm{i}=50 \mu \mathrm{m}$ )

mezcladas a través del radio, escasos biseriados; con altura promedio de los uniseriados de $169 \pm 9 \mu \mathrm{m}(126-220 \mu \mathrm{m})$; con pequeños cuerpos obscuros de forma irregular.

Caracteristicas morfologicas: arbusto o árbol de 6 (3-6) m de altura, ramificación abierta horizontal ascendente; hojas de cada par algo desiguales, suculentas; flores blanco-rosadas; fruto rojizo-verde o morado. Perennifolio.

Nombres comunes: cenizo, patashtillo (Chis.), hojas de lata, manzano, tehuate, teshuate, teshuate blanco, tecalate, yagaguito (Oax.), sabano (Tab.).

Distribución: Jal., Mich., Ver., Gro, Méx., Mor., Pue., Oax., Tab., Chis. 


\section{Myrsine juergensenii (Mez) Ricketson \& Pipoly (MYRSINACEAE) (Fig. $3 \mathrm{~d}-\mathrm{f}$ ).}

Características microscópicas: anillos de crecimiento inconspicuos. Porosidad difusa, vasos de contorno ligeramente anguloso, principalmente solitarios y múltiples radiales de 2 o 3 , en promedio $16 / \mathrm{mm}^{2}$, diámetro tangencial promedio de $69 \pm 4 \mu \mathrm{m}(56-84 \mu \mathrm{m})$; longitud media de los elementos de vaso de $537 \pm 9 \mu \mathrm{m}$ (400-677 $\mu \mathrm{m})$; placa de perforación simple con inclinación de 50-60; punteaduras intervasculares alternas, con diámetro de 3 a $5 \mu \mathrm{m}$; punteaduras vasoradio similares a las intervasculares. Fibras con punteaduras simples, con un septo, con núcleos evidentes; longitud promedio de $989 \pm 18 \mu \mathrm{m}(722-1138 \mu \mathrm{m})$, diámetro total de $23 \pm 4 \mu \mathrm{m}(18-28 \mu \mathrm{m})$, grosor de la pared de 7 a $8 \mu \mathrm{m}$ y diámetro del lumen de $10 \pm 4 \mu \mathrm{m}(6-8 \mu \mathrm{m})$; la relación Runkel es de 1,390, sus fibras son de calidad regular para ser empleadas como pulpa para papel. Parénquima axial paratraqueal vasicéntrico, con series de 2 a 4 células. Radios $2 / \mathrm{mm}$; tipo heterogéneo IIB; no se presentan radios uniseriados; los multiseriados con 7 a 12 células de ancho, formados por células procumbentes en el cuerpo y una hilera de células cuadradas o erectas en los márgenes, existen radios agregados, con altura promedio de $3298 \pm 259 \mu \mathrm{m}$ (1860$6720 \mu \mathrm{m})$ y $199 \pm 38 \mu \mathrm{m}(140-292 \mu \mathrm{m}) \mathrm{de}$ ancho; en las células se observan núcleos y citoplasma evidentes.

Características morfológicas: árbol a veces arbusto de 6-18 $\mathrm{m}$ de altura, dn 27 $\mathrm{cm}$, tronco cilíndrico, derecho o algo tortuoso, irregular en corte transversal; ramas gruesas en copa ovoide a semiesférica. Subperennifolio (Aguilar-Rodríguez et al., 2000).

Nombres comunes: manglillo (Gro.), zapote prieto, chocolatillo (Oax.).
Distribución: Nay., Jal., Méx., Gro., Ver., Oax., Chis.

\section{Phyllonoma laticuspis (Turcz.) Engl. (SAXIFRAGACEAE) (Fig. $3 \mathrm{~g}$-j).}

Características microscópicas: anillos de crecimiento inconspicuos. Porosidad difusa, vasos de contorno anguloso, igual número de vasos solitarios y múltiples radiales de 2 a 7 , en promedio $163 / \mathrm{mm}^{2}$, diámetro tangencial promedio de $36 \pm 6$ $\mu \mathrm{m}$ (28-40 $\mu \mathrm{m})$; longitud media de los elementos de vaso de $907 \pm 25 \mu \mathrm{m}$ (534$1232 \mu \mathrm{m})$; placa de perforación escalariforme con 15 a 20 barras, con inclinación de 60-65\%; punteaduras intervasculares alternas, con diámetro de 4 a $5 \mu \mathrm{m}$; punteaduras vaso-radio similares a las intervasculares. Fibras con punteaduras simples; longitud promedio de $1531 \pm 26$ $\mu \mathrm{m}$ (1138-1876 $\mu \mathrm{m})$, diámetro total de 20 $\pm 4 \mu \mathrm{m}(15-28 \mu \mathrm{m})$, grosor de la pared de 9 a $10 \mu \mathrm{m}$ y diámetro del lumen de $6 \pm 2$ $\mu \mathrm{m}$ (4-10 $\mu \mathrm{m})$; la relación Runkel es de 2,260 , sus fibras son de mala calidad para ser empleadas como pulpa para papel. Parénquima axial raro o ausente. Radios $14 / \mathrm{mm}$; tipo heterogéneo I; los radios son uniseriados de 5 a 15 células de alto, formados por células cuadradas y erectas, con una altura promedio de 1096 \pm 54,43 $\mu \mathrm{m}$ (667-2355 $\mu \mathrm{m})$; ocasionalmente se observan radios biseriados.

Características morfológicas: árbol a veces arbusto de 5 (4-8) $\mathrm{m}$ de altura, ramas lisas y delgadas; hojas alternas lanceoladas, acuminadas y aserradas; flores muy pequeñas de color amarillo a blanquecinas; frutos son como una baya (Martínez, 1979). Perennifolio.

Nombres comunes: guixi-yetza bidao, yaga-lope, hierba de la viruela (Oax.).

Distribución: Ver., Mich., Méx., Gro., Pue., Oax., Chis. 
10. Styrax argenteus C. PresI (sTYRACACEAE) (Fig. 4 a-c).

Características microscópicas: anillos de crecimiento conspicuos, se delimitan por una banda angosta de 4 a 5 hileras de fibras con las paredes comprimidas radialmente en la madera tardía. Porosidad difusa, vasos de contorno ligeramente anguloso, solitarios y múltiples radiales de 2 a 3 , pocos en racimos de 3 y 4 vasos, en promedio $26 / \mathrm{mm}^{2}$, diámetro tangencial promedio de $55 \pm 3 \mu \mathrm{m}(44-76 \mu \mathrm{m})$; longitud media de los elementos de vaso de $1091 \pm 22 \mu \mathrm{m}(785-1345 \mu \mathrm{m})$; placa de perforación escalariforme con 5 a 8 barras, con inclinación de $60-70^{\circ}$; punteaduras intervasculares alternas, con un diámetro de 6 a $7 \mu \mathrm{m}$; punteaduras vaso-radio similares a las intervasculares. Fibras con punteaduras simples, con un septo; longitud promedio de $1347 \pm 26 \mu \mathrm{m}$ (1044$1523 \mu \mathrm{m})$, diámetro total de $17 \pm 4 \mu \mathrm{m}$ (12$26 \mu \mathrm{m})$, grosor de la pared de 4 a $5 \mu \mathrm{m}$ y diámetro del lumen de $9 \pm 3 \mu \mathrm{m}(6-11 \mu \mathrm{m})$; la relación Runkel es de 0,888 , sus fibras son de buena calidad para ser empleadas como pulpa para papel. Parénquima axial vasicéntrico escaso y apotraqueal difuso, con series de más de 8 células. Radios $4 / \mathrm{mm}$; tipo heterogéneo I; los radios uniseriados de 8 a 11 células de alto, formados por células cuadradas y erectas; los multiseriados de 3 a 4 células de ancho, formados por células procumbentes en el cuerpo y más de cuatro hileras de células erectas y/o cuadradas en los márgenes, frecuentemente se unen los márgenes con los otros radios, con altura promedio de $1264 \pm 46 \mu \mathrm{m}(832-1860 \mu \mathrm{m})$ y $46 \pm 6 \mu \mathrm{m}$ $(43-65 \mu \mathrm{m})$ de ancho.

Características morfológicas: arbusto a veces árbol de 15 a $27 \mathrm{~m}$ de altura, dn 15 $\mathrm{cm}$, tronco cilíndrico, circular en corte transversal; ramificación simpódica con ramas horizontales y difusas; copa subpiramidal a irregularmente extendida; hojas pecioladas. Subperennifolio (AguilarRodríguez et al., 2000).

Nombres comunes: capuíin, hoja de abon (Oax.), chucamay (Chis.), chilacuate (Gro.), ruin (Son.), canelillo (Jal.), chilacuate (Mor.), duraznillo, jaboncillo, kuatianu, mamuyo, tapamu (Mich.).

Distribución: Sin., Jal., Mich., Méx., Mor., Ver., Gro., Oax., Chis.

11. Cleyera mexicana (Turcz) Planch. ex Hemsl. (THEACEAE) (Fig. $4 \mathrm{~d}$-f).

Características microscópicas: anillos de crecimiento conspicuos, se delimitan por una banda angosta de 3 a 4 hileras de fibras de paredes más gruesas con lúmenes pequeños en la madera tardía. Porosidad difusa, vasos de contorno anguloso, principalmente solitarios y pocos en grupos de dos, en promedio $63 / \mathrm{mm}^{2}$, diámetro tangencial promedio de $63 \pm 4 \mu \mathrm{m}(48-78 \mu \mathrm{m})$; longitud media de los elementos de vaso de $1714 \pm 45 \mu \mathrm{m}$ (1177-2504 $\mu \mathrm{m})$; placa de perforación escalariforme con 45 a 50 barras, con inclinación de $60^{\circ}$; punteaduras intervasculares opuestas a escalariformes; punteaduras vaso-radio similares a la intervasculares. Fibras con punteaduras areoladas evidentes; longitud promedio de $2572 \pm 46 \mu \mathrm{m}$ (1766-3100 $\mu \mathrm{m})$, diámetro total de $33 \pm 7 \mu \mathrm{m}(25-41 \mu \mathrm{m})$, grosor de la pared de 10 a $11 \mu \mathrm{m}$ y diámetro del lumen de $15 \pm 6 \mu \mathrm{m}(7-22 \mu \mathrm{m})$; la relación Runkel es de 1,187 , sus fibras son de calidad regular para ser empleada como pulpa para papel. Parénquima axial paratraqueal escaso y apotraqueal difuso, con series de más de 8 células; con abundantes contenidos oscuros. Radios $13 / \mathrm{mm}$; tipo heterogéneo I; los radios uniseriados de 10 a 25 células de alto, formados por células cuadradas y erectas; los biseriados, formados por células procumbentes en el cuerpo y de 2 a 4 o más hileras de células erectas y/o 

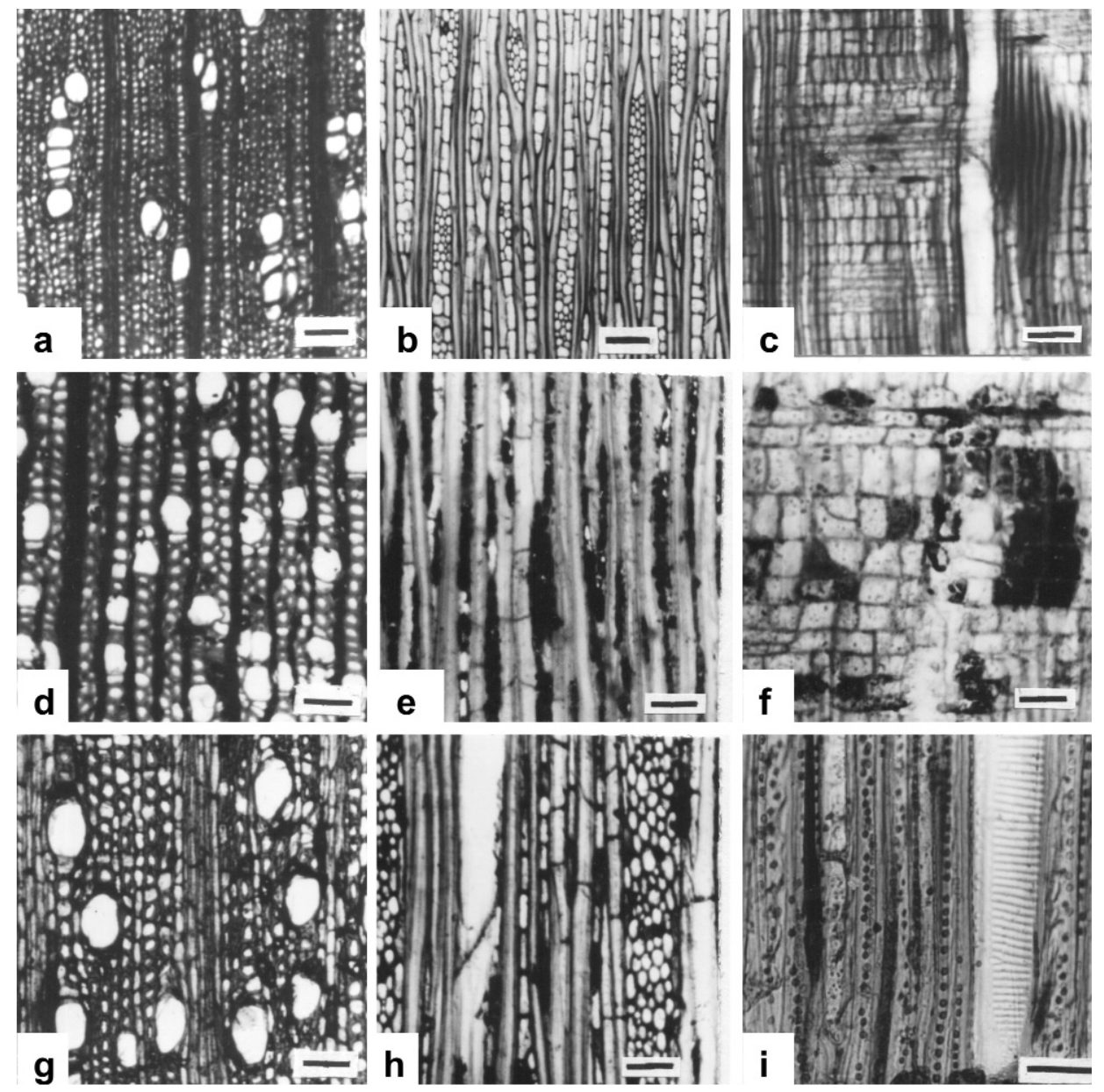

Figura 4. Características microscópicas de la madera.

Cortes transversal (izquierda), tangencial (centro) y radial (derecha). a-c. Styrax argenteus. d-f. Cleyera mexicana. g-i. Ternstroemia pringlei.

(escalas: $a-h=100, \mu \mathrm{m}, \mathrm{i}=50 \mu \mathrm{m}$ )

cuadradas en los márgenes, con altura promedio de $701 \pm 19 \mu \mathrm{m}(479-973 \mu \mathrm{m}) \mathrm{y}$ $28 \pm 4 \mu \mathrm{m}(23-39 \mu \mathrm{m})$ de ancho. Con abundantes contenidos obscuros.

Características morfológicas: árbol de 10 (5-21) m de altura, dn $29 \mathrm{~cm}$, ramificación monopódica; ramas horizontales; copa alargada ovoidal de cobertura media; hojas alternas, elípticas; flores axilares pequeñas. Subperennifolio.

Nombres comunes: escobo (Chis.), capulín de virgen, garambullo, limoncillo, tchcari-charapiti (lengua purépecha), balero (Mich.), trompillo, tilia, blanquito (Méx.), capulín de virgen (Jal.), "tit du juch" (palo verde) (Dgo.). 
Distribución: Sin., Dgo., Jal., Mich., Méx., Gro., Mor., Oax., Chis.

\section{Ternstroemia pringlei (Rose) Standl. (THEACEAE) (Fig. 4 g-i).}

Características microscópicas: anillos de crecimiento conspicuos, se delimitan por una banda angosta de 2 a 3 hileras de fibras con las paredes ligeramente comprimidas radialmente en la madera tardía. Porosidad difusa, vasos de contorno ligeramente anguloso, principalmente solitarios y pocos en grupos de 2 , en promedio $37 / \mathrm{mm}^{2}$, diámetro tangencial promedio de $81 \pm 4 \mu \mathrm{m}(62-94 \mu \mathrm{m})$; longitud media de los elementos de vaso de $1779 \pm 35 \mu \mathrm{m}(1075-2143 \mu \mathrm{m})$; placa de perforación escalariforme con 25 a 40 barras, con inclinación de 50-60 duras intervasculares opuestas, con un diámetro de 6 a $8 \mu \mathrm{m}$; punteaduras vasoradio similares a las intervasculares. Fibras con areolas evidentes, con 1 a 2 septos; longitud promedio de $2795 \pm 46$ $\mu \mathrm{m}(2315-3532 \mu \mathrm{m})$, diámetro total de 17 $\pm 3 \mu \mathrm{m}(13-21 \mu \mathrm{m})$, grosor de la pared de 5 a $6 \mu \mathrm{m}$ y diámetro del lumen de $8 \pm 3$ $\mu \mathrm{m}(5-11 \mu \mathrm{m})$; la relación Runkel es de 1,041 , sus fibras son de calidad regular para ser empleadas como pulpa para papel. Parénquima axial paratraqueal escaso, vasicéntrico y apotraqueal difuso y en agregados, con series de 5 a 8 células. Radios $4 / \mathrm{mm}$; tipo heterogéneo l; los radios uniseriados de 7 a 13 células de alto, formados por células erectas y cuadradas; los multiseriados de 4 a 7 células de ancho y pocos biseriados, formados por células procumbentes en el cuerpo y de 2 a 4 , pocas de 10 , hileras de células erectas y/o cuadradas en los márgenes; con altura promedio de $3776 \pm$ $265 \mu \mathrm{m}(2512-5809 \mu \mathrm{m})$ у $133 \pm 19 \mu \mathrm{m}$ (108-184 $\mu \mathrm{m})$ de ancho.

Características morfológicas: árbol a veces arbusto de 15 (10-20) $\mathrm{m}$ de alto, dn $15 \mathrm{~cm}$, monopódico, ramas irregulares y horizontales, copa irregular; hojas angostamente oblongo-lanceoladas; flores de 5 pétalos; fruto ovoide (Martínez, 1979). Perennifolio.

Nombres comunes: flor de tila, trompillo, palo colorado, cucharillo, hierba del cura, jaboncillo, garra pato (Mich.), taonabo, trompillo, tilia de hoja angosta (Méx.), tila, trompillo (Jal.), limoncillo de monte, tilia (Gro.).

Distribución: Sin., Nay., Jal., Col., Mich., Hgo., Gro., Méx., Mor., Oax., Chis.

\section{DISCUSIÓN}

El estudio anatómico muestra que los anillos de crecimiento en la mayoría de las especies estudiadas sólo se delimitan por bandas de 2 a 6 hileras de fibras con los lúmenes más pequeños y paredes gruesas o comprimidas radialmente en la madera tardía, observándose que esta banda es muy angosta con respecto a la de la madera temprana. En Acer negundo la madera tardía se delimita además por la presencia de algunos vasos de menor diámetro al promedio en esta especie. Sólo en Quercus laurina, Miconia glaberrima, Myrsine juergensenii y Phyllonoma lanticuspis estos anillos no son evidentes o están ausentes. En general, resultó difícil señalar su presencia en varias de las maderas estudiadas y al parecer en los ambientes mesófilos es común que los anillos de crecimiento no se formen claramente. Al respecto, Aguilar-Rodríguez y BarajasMorales (2005) mencionan que en un BMM la presencia o ausencia de estos anillos puede estar relacionada con el clima regional, o con el origen geográfico de las especies.

Los vasos son principalmente solitarios y pequeños, con diámetros menores a $100 \mu \mathrm{m}$, además de ser muy numerosos, más de 21 poros $/ \mathrm{mm}^{2}$ (Chattaway, 
1932). Sólo Quercus laurina e Inga spuria presentan diámetros mayores de $100 \mu \mathrm{m}$ y son pocos (5 y $4 / \mathrm{mm}^{2}$, respectivamente), observándose una relación inversa, ya que conforme aumenta el diámetro de los vasos disminuye su número por $\mathrm{mm}^{2}$. Las placas de perforación son de dos tipos, simples en siete especies y escalariformes en cinco; este último caracter se encuentra frecuentemente en especies que se desarrollan en ambientes húmedos de montaña (AguilarRodríguez y Barajas-Morales, 2005; Versteegh, 1968). Los elementos de vaso y las fibras varían en longitud, de $327 \mu \mathrm{m}$ en Inga spuria a $1779 \mu \mathrm{m}$ en Ternstroemia pringlei y de $523 \mu \mathrm{m}$ en Miconia glaberrima a $2795 \mu \mathrm{m}$ en T. pringlei, respectivamente y de acuerdo a la IAWA (IAWA Committee, 1937) se clasifican como medianos y largos. Sólo en Acer negundo y $M$. glaberrima las fibras son cortas. La tendencia a presentar elementos traqueales medianos y largos es diferente a lo que se observa en los climas secos, en donde estos son principalmente cortos, como se muestra en el trabajo de Abundiz-Bonilla et al. (2004). Nueve especies presentan fibras con lúmenes amplios y paredes delgadas (Chattaway, 1932), excepto Quercus laurina, I. spuria y Phyllonoma lanticuspis, cuyas fibras tienen paredes gruesas y sus lúmenes son angostos. Los valores de las fibras permitieron determinar el grado de calidad para papel de cada especie, observándose que, de acuerdo a la relación de Runkel (Tamarit, 1996), de las doce especies estudiadas, sólo I. spuria y $P$. lanticuspis son de mala calidad.

El parénquima axial es escaso o ausente en 10 especies, variando de apotraqueal difuso a paratraqueal vasicéntrico, sólo en dos es abundante, Quercus laurina e Inga spuria. En los trabajos de Baas (1973), Wheeler y Baas (1991) y Segala y Angyalossy-Alfonso (2002) se reporta que en las maderas de clima templado el parénquima axial es más escaso que en las regiones tropicales. Nuestros resultados concuerdan con los obtenidos por Aguilar-Rodríguez y Barajas-Morales (2005) para las especies de un bosque mesófilo de México. Con respecto a los radios, siete especies presentan el tipo heterogéneo I (Kribs, 1935), que se caracteriza principalmente porque los radios multiseriados usualmente tienen sus márgenes uniseriados muy largos (Carlquist, 1988). Otros tipos encontrados fueron el heterogéneo IIB en Acer negundo y Myrsine juergensenii, homogéneo I en Quercus laurina e Inga spuria y heterogéneo III en Miconia glaberrima. Los tipos de radio de acuerdo a Kribs (1935) ayudan a interpretar aspectos evolutivos de los caracteres anatómicos de las especies, reportándose que los radios tipo heterogéneo I están relacionados con maderas primitivas (Carlquist, 1988).

Los caracteres de Cornus disciflora, Quercus laurina, Styrax argenteus, Ternstroemia pringlei y Xylosma flexuosa, se compararon con los reportados por Aguilar-Rodríguez et al. (2000), De la Paz Pérez et al. (1982), López (1997) y Quintanar et al. (1996) para los mismos taxa (Tabla 3). Se observó variación en las dimensiones de los elementos traqueales y los radios. En otros trabajos se ha reportado que entre individuos de una misma especie, estas variaciones frecuentemente están influenciadas por algunos aspectos ambientales, como la humedad y la temperatura, así como por el hábito de las especies (Aguilar-Rodríguez et al., 2006; Metcalfe y Chalk, 1983), ya que la longitud de los elementos de vaso y las fibras, así como la altura de los radios aumentan sus dimensiones conforme se incrementa la altura de los individuos (Aguilar-Rodríguez, 2001; Terrazas, 1994). Con respecto a los caracteres cualitativos, las punteaduras intervasculares de los elementos de vaso 
de Ternstroemia pringlei se observan opuestas, como también lo reportan Aguilar-Rodríguez et al. (2000) para esta especie. Sin embargo, no coincidimos con De la Paz Pérez et al. (1982), en donde las reportan como areoladas alternas. En el caso de Quercus laurina aquí se describe con un parénquima axial vasicéntrico y escalariforme en bandas angostas de 1 a 3 células de ancho, mientras que Aguilar-Rodríguez et al. (2000) lo reportan como paratraqueal y apotraqueal difuso en agregados con tendencia a ser diagonal. Estas diferencias cuantitativas y cualitativas encontradas sugieren que se intensifiquen los estudios anatómicos de la madera sobre variabilidad intraespecífica.

\section{CONCLUSIONES}

Los caracteres anatómicos de la madera de las especies estudiadas presentan maderas con anillos de crecimiento formados por bandas angostas de fibras con lúmenes reducidos y paredes gruesas en la madera tardía, porosidad difusa, elementos de vaso y fibras medianas y largas, vasos numerosos con diámetros pequeños y placas de perforación escalariforme en varias especies; parénquima axial en general escaso y radios heterogéneos tipo I. La comparación de especies descritas en otros trabajos con las mismas estudiadas aquí revelaron que existen diferencias cuantitativas y/o cualitativas en Cornus disciflora, Quercus laurina, Styrax argenteus, Ternstroemia pringlei y Xylosma flexuosa. Estas variaciones pueden deberse a las características propias del ambiente donde los individuos crecen, así como a su altura o hábito de crecimiento.

\section{RECONOCIMIENTOS}

Agradecemos al Dr. J. Daniel TejeroDíez, del laboratorio de Botánica, de la
Unidad de Morfología y Función de la FES Iztacala UNAM, por la donación de algunas muestras de madera y la identificación de las especies, así como por sus valiosas sugerencias para mejorar este manuscrito.

\section{REFERENCIAS}

Abundiz-Bonilla, L.A.M. 1988. El género Selaginella Beauv. en la parte oeste del Estado de México. Tesis licenciatura. Escuela Nacional de Estudios Profesionales Iztacala, Universidad Nacional Autónoma de México. Tlalnepantla, Estado de México. 85 p.

Abundiz-Bonilla, L.A.M., J. BarajasMorales y P. Tenorio-Lezama. 2004. Anatomía de maderas de México. Árboles y arbustos del matorral xerófilo de Tehuacán, Puebla. Instituto de Biología. Publicaciones Especiales 19. Universidad Nacional Autónoma de México. México. $97 \mathrm{p}$.

Aguilar-Rodríguez, S. 2001. Anatomía de la madera de Buddleja L. y $B$. cordata H.B.K. (Buddlejaceae): Un enfoque ecológico-filogenético. Tesis de Doctorado. Colegio de Postgraduados, Montecillo,Texcoco. México. $131 \mathrm{p}$.

Aguilar-Rodríguez, S y J. BarajasMorales. 2005. Anatomía de la madera de especies arbóreas de un bosque mesófilo de montaña: un enfoque ecológico-evolutivo. Boletín de la Sociedad Botánica de México 77: 51-59.

Aguilar-Rodríguez, S., J. Barajas-Morales y D. Tejero-Díez. 2000. Anatomía de maderas de México: especies de un bosque mesófilo de montaña. Instituto de Biología. Publicaciones especiales 17. Universidad Nacional Autónoma de México. México. 87 p. 
Aguilar-Rodríguez, S., T. Terrazas y L. López-Mata. 2006. Anatomical wood variation of Buddleja cordata (Buddlejaceae) along its natural range in Mexico. Trees 20(2): 253-261.

Baas, P. 1973. The wood anatomical range in Ilex (Aquifoliaceae) and its ecological and phylogenetic significance. Blumea 21: 193-258.

Barajas-Morales, J. 1980. Anatomía de maderas de México núm. 3. Diez especies del bosque caducifolio de las cercanías de Xalapa, Veracruz. México. Biotica 5(1): 23-40.

Berlyn, G.P. y J.P. Miksche. 1976. Botanical microtechnique and cytochemistry. lowa State University Press. lowa. 326 p.

Carlquist, S. 1988. Comparative wood anatomy. Systematic, ecological, and evolutionary aspects of dicotyledon wood. Springer-Verlag, Berlin. $436 \mathrm{p}$.

Carmona V.,F. 1979. Características histológicas de la madera de cuatro especies del bosque caducifolio de México. Tesis licenciatura. Facultad de Ciencias. Universidad Nacional Autónoma de México. México, D.F. $170 \mathrm{p}$.

Chattaway, M.M. 1932. Proposed standards for numerical values used in describing woods. Tropical Woods 29: $20-28$.

De la Paz Pérez O., C. 1976. Características anatómicas de cinco encinos de México. Boletín Técnico del Instituto Nacional de Investigaciones Forestales núm. 46. México. 43 p.

De la Paz Pérez O., C. 1980. Estudio anatómico de la madera de once especies de angiospermas. Boletín
Técnico del Instituto Nacional de Investigaciones Forestales No. 64. México. $79 \mathrm{p}$.

De la Paz Pérez O., C. 1982. Estructura anatómica de cinco especies del género Quercus. Boletín Técnico del Instituto Nacional de Investigaciones Forestales núm. 88. México. 62 p.

De la Paz Pérez O., C., P. Olvera C. y G. Corral L. 1982. Estudio anatómico de la madera de 26 especies de angiospermas de clima templado. Boletín Técnico del Instituto Nacional de Investigaciones Forestales núm. 91. México. $123 \mathrm{p}$.

Fragoso-Ramírez, R. 1990. Estudio florístico en la parte alta de la Sierra de Zacualpa, Estado de México. Tesis de Llicenciatura. Escuela Nacional de Estudios Profesionales Iztacala, Universidad Nacional Autónoma de México. Tlalnepantla, Estado de México. 82 p.

Guridi, L. y A. García. 1997. Las maderas en los instrumentos musicales de cuerda de Paracho. Ediciones Casa Hidalgo. México. 45 p.

Huerta-Crespo, J. 1975. 100 maderas mexicanas. Instituto Nacional de Investigaciones Forestales, Publicaciones especiales núm. 9. México.

IAWA Committee. 1937. Committee on the standardization of terms of cell size. Standard terms of length of vessel members and wood fiber. Tropical Woods 51: 21-22.

IAWA Committee. 1939. Committee on the nomenclature. Standard terms of size for vessel diameter and ray width. Tropical Woods 59: 51-52.

IAWA Committee. 1989. IAWA list of microscopic features for hardwood identifi- 
cation. International Association of Wood Anatomist Bulletin new series 10 (3): 219-332.

Johansen, J. 1940. Plant microtechnique. McGraw-Hill Book Company, Nueva York. 523 p.

Kribs, D.A. 1935. Salient lines of specialization in the wood rays of dicotyledons. Botanical Gazetle 96 (1):547557.

López, M.S. 1997. Tendencias ecológicas en la estructura de la madera de árboles de un bosque mesófilo de montaña de Michoacán. Tesis de Licenciatura. Facultad de Estudios Superiores Iztacala, Universidad Nacional Autónoma de México. Tlalnepantla, Estado de México. 80 p.

López-Pérez, Y. 1995. Inventario florístico y conocimiento estructural del bosque mesófilo en el municipio de Valle de Bravo, Estado de México. Tesis de Licenciatura. Campus Iztacala, Universidad Nacional Autónoma de México. Tlalnepantla, Estado de México. 92 p.

Luna-Vega, I., L. Almeida-Leñero y J. Llorente-Bousquets. 1989. Florística y aspectos fitogeográficos del bosque mesófilo de montaña de las Cañadas de Ocuilán, estados de Morelos y México. Anales del Instituto de Biología. Universidad Nacional Autónoma de México, Serie Botánica 59 (1): 63-87.

Martínez, M. 1979. Catálogo de nombres vulgares y científicos de plantas mexicanas. Fondo de Cultura Económica. México. 857 p.

Metcalfe, C.R. y L. Chalk. 1983. Anatomy of the dicotyledons. 2nd edition. Vol. II. Clarendon Press. Oxford. 315 p.
Miranda, F. 1947. Estudios sobre la vegetación de México. V: Rasgos de la vegetación de la Cuenca del Río Balsas. Revista Sociedad Mexicana. Historia Natural. Tomo VIII,1(4):95114.

Miranda, J. M y M.A. González O. 1993. Estudio de la vegetación y florística de la Mesa Basáltica de Holotepec, Distrito de Tenango del Valle, Estado de México. Tesis de Licenciatura. Escuela Nacional de Estudios Profesionales Iztacala, Universidad Nacional Autónoma de México. Tlalnepantla, Estado de México. 74 p.

Orozco, M.V. 1995. Vegetación del municipio de Temascaltepec, Estado de México. Tesis de Licenciatura. Campus Iztacala, Universidad Nacional Autónoma de México. Tlalnepantla, Estado de México. 100 p.

Ortega E., F. y G. Castillo C. 1996. El bosque mesófilo de montaña y su importancia forestal. Ciencias (43): 32-39.

Quintanar, A., C. De la Paz Pérez O., I. de la Cruz y D. Razo. 1996. Anatomía de la madera de ocho especies de angiospermas de clima templado. Boletín de la Sociedad Botánica (58): 5-14.

Rzedowski, J. y G. Calderón de Rzedowski. 1985. Flora fanerogámica del Valle de México. Escuela Nacional de Ciencias Biológicas, Instituto Politécnico Nacional. Instituto de Ecología. México, D.F. Vol. II. $674 \mathrm{p}$.

Rzedowski, J. 1986. Vegetación de México. Limusa. México. 432 p.

Rzedowski, J. 1996. Análisis preliminar de la flora vascular de los bosques 
mesófilos de montaña de México. Acta Botánica Mexicana (35): 25-44.

Sánchez, G.N. 1968. Estudio estadístico de la variación de la longitud, el grosor y el diámetro de las fibras leñosas de Fraxinus udhei Wenzing. Tesis de Licenciatura. Facultad de Ciencias. Universidad Nacional Autónoma de México. México, D.F. $101 \mathrm{p}$.

Segala, A. E. y V. Angyalossy-Alfonso. 2002. Ecological trends in the wood anatomy of some brazilian species. 2. axial parenchyma, rays and fibres. IAWA Journal 23(4):391-418.

Tamarit U., J.C. 1996. Determinación de los índices de calidad de pulpa para papel de 132 maderas latifoliadas. Madera y Bosques 2(2):29-41.

Terrazas, T. 1994. Wood anatomy of Anacardiaceae: ecological and phylogenetic interpretation. Doctoral Dissertation University of North Carolina-Chapel Hill. 321 p.

Tomalang, F. N. y F. F. Wangaard. 1961. Relationships between hardwood fiber characteristics and pulpsheet properties. Tappi 44(3):201-216.
Torres, P. 1982. Maderas utilizadas en la fabricación de instrumentos musicales de cuerda en la Huasteca. Tesis de Licenciatura. Facultad de Ciencias. Universidad Nacional Autónoma de México. México. $109 \mathrm{p}$.

Torres-Zúñiga, M.M. y J.D. Tejero-Díez. 1998. Flora y vegetación de la sierra de Sultepec, Estado de México. Anales del Instituto de Biología Universidad Nacional Autónoma de México, Serie Botánica 69(2):135174.

Versteegh, C. 1968. Anatomical study of some woody plants of mountain flora in the tropics (Indonesia). Acta Botanica Neerlandica 17:151-159.

W3 Tropicos. 2005. Missouri Botanical Garden's VAST nomenclatural database and associated authority files. <http://mobot.mobot.org/W3T/Searc h/vast.html>

Wheeler, E.A. y P. Baas. 1991. A survey of the fossil record for dicotyledonous wood and its significance for evolutionary and ecological wood anatomy. IAWA Bulletin new series 12(3):275-332. 